\title{
BASIC HYPERGEOMETRIC SUMMATIONS FROM ROOK THEORY
}

\author{
MICHAEL J. SCHLOSSER* AND MEESUE YOO** \\ Dedicated to Professor Krishnaswami Alladi on the occasion of his 60th birthday
}

\begin{abstract}
We employ a one-variable extension of $q$-rook theory to give combinatorial proofs of some basic hypergeometric summations, including the $q$-Pfaff-Saalschütz summation and a ${ }_{4} \phi_{3}$ summation by Jain.
\end{abstract}

\section{INTRODUCTION}

The theory of $q$-series has a prominent history. It made its first appearance in a combinatorial study by Euler on partitions of numbers. Among the first $q$-series identities were explicit summations for two different $q$-analogues of the exponential series. These identities were later unified by Gauß, Heine, and Cauchy who, all three independent from each other, discovered and proved the nonterminating $q$-binomial theorem. This initiated the systematic study of $q$-hypergeometric series, or synonymously, basic hypergeometric series, "basic" referring to the base $q$, as objects of their own interest, separate from combinatorics. While in the early days only a small number of mathematicians studied the combinatorics of $q$-series (most notably, J. J. Sylvester in the 19th century, and P. A. MacMahon and I. Schur in the early 20th century, to name just a few figures whose research had big impact) the situation rapidly changed in the 1960s when B. Gordon found extensions of the Rogers-Ramanujan identities with combinatorial interpretations, after which many more people entered the scene. See Andrews' book chapter [1] for an account of the history of $q$-series and partitions. Further, the preface of Gasper and Rahman's textbook [5] provides a brief history of basic hypergeometric series, and the book itself contains further background on the subject.

Basic hypergeometric series appear from time to time in combinatorial studies. It is particularly instructive to see combinatorial proofs of $q$-series identities. Having a combinatorial interpretation of an identity at hand leads to a better understanding, since one gets a feeling why the identity is true. Now, focusing on combinatorial interpretations and given a reasonably simple identity, it is by all means legitimate to ask the question: is there a combinatorial proof for it? For instance, for the well-known $q$-Pfaff-Saalschütz summation,

$$
\sum_{k=0}^{n} \frac{\left(a, b, q^{-n} ; q\right)_{k}}{\left(q, c, a b q^{1-n} / c ; q\right)_{k}} q^{k}=\frac{(c / a, c / b ; q)_{n}}{(c, c / a b ; q)_{n}},
$$

(see Section 2 for the notation) several combinatorial proofs are known [2, 8, 9, 16, 21, 22, But what about a combinatorial proof of the following summation by Jain [12] (which is a

\footnotetext{
2010 Mathematics Subject Classification. Primary 05A19; Secondary 05A15, 05A30, 11B65, 11B73, 11 B83.

Key words and phrases. rook numbers, $q$-analogues, alpha-parameter model, matchings.

*Partly supported by FWF Austrian Science Fund grant F50-08 within the SFB "Algorithmic and enumerative combinatorics".

${ }^{* *}$ Fully supported by FWF Austrian Science Fund grant F50-08 within the SFB "Algorithmic and enumerative combinatorics".
} 
$q$-analogue of a ${ }_{3} F_{2}$ summation by Bailey [3] and can be written as a summation for a specific ${ }_{4} \phi_{3}$ series)?

$$
\sum_{k=0}^{n} \frac{(a, b ; q)_{k}\left(q^{-2 n} ; q^{2}\right)_{k}}{\left(q, q^{-2 n} ; q\right)_{k}\left(a b q ; q^{2}\right)_{k}} q^{k}=\frac{\left(a q, b q ; q^{2}\right)_{n}}{\left(q, a b q ; q^{2}\right)_{n}}
$$

In this paper we present a new combinatorial proof of (1.1) and also, to the best of our knowledge, a first combinatorial proof of (1.2), in addition to a few similar results. We achieve this by employing a specific one-variable extension of $q$-rook theory with extra variable $a$, which we shall refer to as $(a ; q)$-rook theory for short. (The $(a ; q)$-rook numbers we are dealing with in this paper are actually special cases of the elliptic rook numbers which we recently have considered in [18, 19, 20. While the elliptic rook numbers satisfy nice identities, they don't factorize in general. However, for particular boards the $(a ; q)$-rook numbers do factorize into closed form. This is the reason why we focus on the $(a ; q)$-case here which is still more general than the $q$-case.)

Already earlier, Haglund [10] has made out intimate connections between rook theory and (basic) hypergeometric series. In particular, he showed that a big class of $(q$-)rook numbers generally admit a representation in terms of (basic) hypergeometric series of Karlsson-Minton type. In our case, we are on one hand (for three different rook models) working with $(a ; q)$ rook numbers, i.e., we add an extra parameter to the $q$-rook numbers. On the other hand, we are looking at very special situations, obtained by restricting to special boards, where the $(a ; q)$-rook numbers nicely factorize. Since the $(a ; q)$-rook numbers satisfy certain product formulas, we are thus able to obtain explicit summations, by substituting the factorized forms in the product formulas.

In Section 2 we recall standard $q$-series notation and introduce the special $(a ; q)$-weights that we use. Section 3 is devoted to $(a ; q)$-rook theory. We explain all the ingredients we need for the $(a ; q)$-extensions of the different rook models that we work with, namely, the standard model, the (more general) alpha-parameter model, and the matching model. From these we deduce basic hypergeometric summations as applications.

\section{Standard $q$-NOTATION AND $(a ; q)$-Weights}

For a parameter $q$, called the base, and variable $u$, the $q$-shifted factorial is defined by

$$
(u ; q)_{0}=1, \quad \text { and } \quad(u ; q)_{n}=(1-u)(1-u q) \ldots\left(1-u q^{n-1}\right) .
$$

(The index $n$ can also be $\infty$, then the product is an infinite product in which case one requires $|q|<1$, for convergence.) For brevity, we frequently use the notation

$$
\left(a_{1}, \ldots, a_{m} ; q\right)_{n}=\left(a_{1} ; q\right)_{n} \ldots\left(a_{m} ; q\right)_{n} .
$$

The $q$-number of $z$ is defined as

$$
[z]_{q}=\frac{1-q^{z}}{1-q} .
$$

We now introduce $(a ; q)$-weights which include an additional variable $a$. Define

$$
\begin{gathered}
w_{a ; q}(k)=\frac{\left(1-a q^{2 k+1}\right)}{\left(1-a q^{2 k-1}\right)} q^{-1}, \\
W_{a ; q}(k)=\frac{\left(1-a q^{2 k+1}\right)}{(1-a q)} q^{-k}, \\
{[z]_{a ; q}=\frac{\left(1-q^{z}\right)\left(1-a q^{z}\right)}{(1-q)(1-a q)} q^{1-z},}
\end{gathered}
$$


for any value $k$, which we call the small weights, big weights, and the $(a ; q)$-number of $z$, respectively. Note that in the limit case $a \rightarrow \infty$, we recover the $q$-weights

$$
\lim _{a \rightarrow \infty} w_{a ; q}(k)=q, \quad \lim _{a \rightarrow \infty} W_{a ; q}(k)=q^{k}, \quad \lim _{a \rightarrow \infty}[z]_{a ; q}=\frac{1-q^{z}}{1-q}=[z]_{q} .
$$

For a positive integer $k$, we have

$$
W_{a ; q}(k)=\prod_{i=1}^{k} w_{a ; q}(i)
$$

Other useful properties are

$$
[y+z]_{a ; q}=[y]_{a ; q}+W_{a ; q}(y)[z]_{a q^{2 y} ; q},
$$

and

$$
W_{a ; q}(k+n)=W_{a ; q}(k) W_{a q^{2 k} ; q}(n) .
$$

Remark 2.1. This $(a ; q)$-weight was first defined in [17] to generalize the binomial theorem for noncommuting variables. That is, in the unital algebra $\mathbb{C}_{a ; q}[x, y]$ over $\mathbb{C}$ defined by the following commutation relations

$$
\begin{aligned}
& y x=\frac{\left(1-a q^{3}\right)}{(1-a q)} q^{-1} x y, \\
& x a=q a x, \\
& y a=q^{2} a y,
\end{aligned}
$$

the binomial theorem

$$
(x+y)^{n}=\sum_{k=0}^{n}\left[\begin{array}{l}
n \\
k
\end{array}\right]_{a ; q} x^{k} y^{n-k}
$$

holds, where the $(a ; q)$-binomial coefficients are defined by

$$
\left[\begin{array}{c}
n \\
k
\end{array}\right]_{a ; q}:=\frac{\left(q^{1+k}, a q^{1+k} ; q\right)_{n-k}}{(q, a q ; q)_{n-k}} q^{k(k-n)}=\frac{[n]_{a ; q} !}{[k]_{a ; q} ![n-k]_{a ; q} !},
$$

with the $(a ; q)$-factorials being defined by

$$
[0]_{a ; q} !=1, \quad[n]_{a, q} !=[n]_{a, q}[n-1]_{a, q} ! .
$$

The $(a ; q)$-binomial coefficients are symmetric in $(k, n-k)$ (whereas the more general elliptic extension of (2.1), considered in [16, 17], is not). They satisfy the two recursions

$$
\begin{aligned}
& {\left[\begin{array}{c}
n+1 \\
k
\end{array}\right]_{a ; q}=\left[\begin{array}{l}
n \\
k
\end{array}\right]_{a ; q}+\frac{\left(1-a q^{2 n+2-k}\right)}{\left(1-a q^{k}\right)} q^{k-n-1}\left[\begin{array}{c}
n \\
k-1
\end{array}\right]_{a ; q},} \\
& {\left[\begin{array}{c}
n+1 \\
k
\end{array}\right]_{a ; q}=\frac{\left(1-a q^{n+1+k}\right)}{\left(1-a q^{n+1-k}\right)} q^{-k}\left[\begin{array}{c}
n \\
k
\end{array}\right]_{a ; q}+\left[\begin{array}{c}
n \\
k-1
\end{array}\right]_{a ; q},}
\end{aligned}
$$

which, together with the initial conditions

$$
\left[\begin{array}{l}
0 \\
0
\end{array}\right]_{a ; q}=1, \quad \text { and } \quad\left[\begin{array}{l}
n \\
k
\end{array}\right]_{a ; q}=0, \quad \text { for } k>n \text { or } k<0,
$$

determine them uniquely. 


\section{3. $(a ; q)$-ROOK THEORY}

For an introduction to classical rook theory, see [4]. A lot of material on generalized rook theory which we survey is borrowed from our papers [18, 19, 20] on elliptic rook theory. As mentioned in the introduction, the $(a ; q)$-case is just a special case of the elliptic case which admits particularly attractive closed formulas. We utilize the closed formulas from the $(a ; q)$ extensions of different rook models to derive some concrete basic hypergeometric summations.

3.1. $(a ; q)$-Extension of the standard model. Let $\mathbb{N}$ denote the set of positive integers. We consider a finite subset of the $\mathbb{N} \times \mathbb{N}$ grid which we refer to as a board, and label the columns and rows by $1,2, \ldots$, from the left and from the bottom, respectively. We use $(i, j)$ to denote the cell in the intersection of the column $i$ and the row $j$.

Let $B\left(b_{1}, b_{2}, \ldots, b_{n}\right)$ denote the set of cells

$$
B=B\left(b_{1}, \ldots, b_{n}\right)=\left\{(i, j) \mid 1 \leq i \leq n, 1 \leq j \leq b_{i}\right\},
$$

for nonnegative integer $b_{i}$ 's, for all $i$. If a board $B$ can be represented by the set $B\left(b_{1}, \ldots, b_{n}\right)$ with nondecreasing integer sequence $0 \leq b_{1} \leq \cdots \leq b_{n}$, then the board $B=B\left(b_{1}, \ldots, b_{n}\right)$ is called a Ferrers board. Given a Ferrers board, we say that we place $k$ nonattacking rooks in $B$ by choosing a $k$-subset of $B$ such that no two elements have a common coordinate. Let $\mathcal{N}_{k}(B)$ denote the set of all nonattacking placements of $k$ rooks in $B$. Note that $\left|\mathcal{N}_{k}(B)\right|$ is the original $k$-th rook number defined in [13].

Given a rook placement $P \in \mathcal{N}_{k}(B)$, a rook in $P$ is said to cancel all the cells to the right in the same row and all the cells below it in the same column. Let $U_{B}(P)$ denote the set of cells in $B-P$ which are not cancelled by any rook in $P$. We define the $(a ; q)$-analogue of the $k$-th rook number by assigning the small weights $w_{a ; q}(j)$ to the respective cells in $U_{B}(P)$, depending on their position and the configuration of rooks.

Definition 3.1. Given a Ferrers board $B=B\left(b_{1}, \ldots, b_{n}\right)$, let the $k$ th $(a ; q)$-rook number be

$$
r_{k}(a, q ; B)=\sum_{P \in \mathcal{N}_{k}(B)} w t(P),
$$

where

$$
w t(P)=\prod_{(i, j) \in U_{B}(P)} w_{a ; q}\left(i-j-r_{(i, j)}(P)\right),
$$

and $r_{(i, j)}(P)$ counts the number of rooks in $P$ positioned in the north-west region of $(i, j)$.

This $(a ; q)$-analogue of the rook numbers satisfy the following product formula which was proved with original rook numbers, i.e., in the $a \rightarrow \infty, q \rightarrow 1$ case, by Goldman, Joichi, and White [7].

Theorem 3.2 ([18]). For any Ferrers board $B=B\left(b_{1}, \ldots, b_{n}\right)$, we have

$$
\prod_{i=1}^{n}\left[z+b_{i}-i+1\right]_{a q^{2\left(i-1-b_{i}\right) ; q}}=\sum_{k=0}^{n} r_{n-k}(a, q ; B) \prod_{j=1}^{k}[z-j+1]_{a q^{2(j-1)} ; q} .
$$

Remark 3.3. In [18, we prove Theorem 3.2 with more general rook numbers. That is, the rook numbers $r_{k}(a, b ; q, p ; B)$ and the weights used to define $r_{k}$ in (3.1) are elliptic (i.e., meromorphic and doubly-periodic) and include two more parameters $b$ and $p$. The $(a ; q)$-rook numbers can be obtained from the elliptic ones by letting $p \rightarrow 0$ and $b \rightarrow 0$.

By distinguishing the cases when there is a rook or not in the last column, we obtain a recursion for the $(a ; q)$-rook numbers. 
Proposition 3.4. Let $B$ be a Ferrers board with $l$ columns of height at most $m$, and $B \cup m$ denote the board obtained by adding the $(l+1)$-st column of height $m$ to the right of $B$. Then, for integer $k$ with $1 \leq k \leq l+1$, we have

$$
r_{k}(a, q ; B \cup m)=W_{a q^{2(l-m)} ; q}(m-k) r_{k}(a, q ; B)+[m-k+1]_{a q^{2(l-m)} ; q} r_{k-1}(a, q ; B),
$$

assuming the conditions

$$
\begin{array}{ll}
r_{k}(a, b ; q, p ; B)=0 & \text { for } k<0 \text { or } k>l, \text { and } \\
r_{0}(a, b ; q, p ; B)=1 & \text { for } l=0, \text { i.e. for } B \text { being the empty board. }
\end{array}
$$

In the case of a rectangular shape board $B=[l] \times[m]$, where $[n]:=\{1,2, \ldots, n\}$, the $(a ; q)$-rook number has a closed form expression which can be proved by the recursion in Proposition 3.4

$$
r_{k}(a, q ;[l] \times[m])=q^{\left(\begin{array}{c}
k+1 \\
2
\end{array}\right)-l m}\left[\begin{array}{l}
l \\
k
\end{array}\right]_{q} \frac{[m]_{q} !}{[m-k]_{q} !} \frac{\left(a q^{l-m-k} ; q\right)_{k}\left(a q^{1+2 l-2 m} ; q^{2}\right)_{m-k}}{\left(a q^{1-2 m} ; q^{2}\right)_{m}} .
$$

For more details, including the omitted proofs, see [18].

3.1.1. $r$-Restricted Lah numbers. The $r$-restricted Lah numbers count the number of placements of the elements $1,2, \ldots, n$ into $k$ nonempty tubes of linearly ordered elements such that $1,2, \ldots, r$ are in distinct tubes (cf. [15], or [14]). These numbers admit a rook theoretic interpretation when $B$ is the board $\mathrm{L}_{n}^{(r)}=[n+r-1] \times[n-r]$. In [18, Subsection 3.4], we have established a correspondence between the rook configurations $P$ of $n-k$ nonattacking rooks on $\mathrm{L}_{n}^{(r)}$ and the set of placements $T$ of the elements $1,2, \ldots, n$ into $k$ nonempty tubes of linearly ordered elements such that the first $r$ numbers $1,2, \ldots, r$ are in distinct tubes. For the full description of the correspondence, refer to [18].

For the Ferrers board $B=\mathrm{L}_{n}^{(r)}$, the product formula in Theorem 3.2 becomes

$$
\begin{aligned}
& \prod_{i=1}^{n-r}[z+n-i]_{a q^{2(i-n)} ; q} \prod_{i=1}^{r}[z-i+1]_{a q^{2(i-1)} ; q} \\
& =\sum_{k=r}^{n} r_{n-k}\left(a q^{2(1-r)}, q ; \mathrm{L}_{n}^{(r)}\right) \prod_{j=1}^{k}[z-j+1]_{a q^{2(j-1)} ; q},
\end{aligned}
$$

after doing certain shifts of variables and cancellation of factors. We define an $(a ; q)$-analogue of the $r$-restricted Lah numbers by

$$
\mathcal{L}_{n, k}^{(r)}(a, q):=r_{n-k}\left(a q^{2(1-r)}, q ; \mathrm{L}_{n}^{(r)}\right) .
$$

It can be shown that $\mathcal{L}_{n, k}^{(r)}(a, q)$ satisfy the following recursion

$$
\mathcal{L}_{n+1, k}^{(r)}(a, q)=W_{a q^{-2 n} ; q}(n+k-1) \mathcal{L}_{n, k-1}^{(r)}(a, q)+[n+k]_{a q^{-2 n} ; q} \mathcal{L}_{n, k}^{(r)}(a, q),
$$

assuming the initial conditions

$$
\begin{aligned}
\mathcal{L}_{n, k}^{(r)}(a, q)=0 & \text { for } k<r-1 \text { or } k>n, \\
\mathcal{L}_{r-1, r-1}^{(r)}(a, q)=1 & \text { (an artificial but felicious initial condition). }
\end{aligned}
$$


Since the board $\mathbf{L}_{n}^{(r)}$ is of rectangular shape, (3.2) gives the closed form formula for $\mathcal{L}_{n, k}^{(r)}(a, q)$, namely,

$$
\mathcal{L}_{n, k}^{(r)}(a, q)=q^{\left(\begin{array}{c}
k \\
2
\end{array}\right)-\left(\begin{array}{c}
n \\
2
\end{array}\right)-n(k-1)+2\left(\begin{array}{c}
r \\
2
\end{array}\right)}\left[\begin{array}{l}
n+r-1 \\
k+r-1
\end{array}\right]_{q} \frac{[n-r]_{q} !}{[k-r]_{q} !} \frac{\left(a q^{1-n+k} ; q\right)_{n-k}\left(a q^{1+2 r} ; q^{2}\right)_{k-r}}{\left(a q^{3-2 n} ; q^{2}\right)_{n-r}} .
$$

Combining (3.4) with the product formula (3.3) gives a combinatorial proof of the $q$-PfaffSaalschütz sum, in the following form :

\section{Proposition 3.5.}

$$
\frac{\left(q^{z+r} ; q\right)_{n}\left(a^{-1} q^{r-z} ; q\right)_{n}}{\left(a^{-1} ; q\right)_{n}\left(q^{2 r} ; q\right)_{n}}=\sum_{k=0}^{n} \frac{\left(q^{-n} ; q\right)_{k}\left(q^{r-z} ; q\right)_{k}\left(a q^{z+r} ; q\right)_{k}}{(q ; q)_{k}\left(q^{2 r} ; q\right)_{k}\left(a q^{1-n} ; q\right)_{k}} q^{k}
$$

Proof. If we replace $r_{n-k}\left(a q^{2(1-r)}, q ; \mathrm{L}_{n}^{(r)}\right)$ by the closed form given in (3.4), we obtain

$$
\begin{aligned}
& \frac{\left(q^{z+r} ; q\right)_{n-r}\left(a q^{z-n+1} ; q\right)_{n-r}}{(1-q)^{n-r}\left(a q^{3-2 n} ; q^{2}\right)_{n-r}} \frac{\left(q^{z-r+1} ; q\right)_{r}\left(a q^{z} ; q\right)_{r}}{(1-q)^{r}\left(a q ; q^{2}\right)_{r}} q^{-n z+\frac{1}{2} n(3-n)+r^{2}-r} \\
&=\sum_{k=r}^{n} q^{\left(\begin{array}{c}
k \\
2
\end{array}\right)-\left(\begin{array}{c}
n \\
2
\end{array}\right)-n(k-1)+2\left(\begin{array}{c}
r \\
2
\end{array}\right)-k z+\left(\begin{array}{c}
k+1 \\
2
\end{array}\right)} \frac{(q ; q)_{n+r-1}}{(q ; q)_{n-k}(q ; q)_{k+r-1}} \frac{(q ; q)_{n-r}}{(q ; q)_{k-r}(1-q)^{n-k}} \\
& \times \frac{\left(q^{z-k+1} ; q\right)_{k}\left(a q^{z} ; q\right)_{k}}{(1-q)^{k}\left(a q ; q^{2}\right)_{k}} \frac{\left(a q^{1-n+k} ; q\right)_{n-k}\left(a q^{1+2 r} ; q^{2}\right)_{k-r}}{\left(a q^{3-2 n} ; q^{2}\right)_{n-r}} .
\end{aligned}
$$

Then (3.5) is the result of simplifying (3.6) with appropriate shifts of $n$ and $k$.

Remark 3.6. If we perform the substitution $A=a q^{z+r}, B=q^{r-z}$ and $C=q^{2 r}$ in (3.5), we get

$$
\frac{(C / A, C / B ; q)_{n}}{(C, C / A B ; q)_{n}}={ }_{3} \phi_{2}\left[\begin{array}{l}
A, B, q^{-n} \\
C, A B C^{-1} q^{1-n} ; q, q
\end{array}\right]
$$

which is the $q$-Pfaff-Saalschütz summation [5, (II.12)], written in standard basic hypergeometric form (cf. [5]). The problem with this substitution is that whereas $a$ and $z$ are general parameters, $r$ is not. To show that (3.5), where $r$ is a nonnegative integer, is actually equivalent to the general case where $r$ is any complex number, works by a standard polynomial argument. If we multiply both sides of (3.5) by $\left(q^{2 r} ; q\right)_{n}$ and formally replace $q^{r}$ by $x$ we obtain a polynomial equation in $x$ of degree $2 n$ which is valid for $x=q^{r}$, for $r=0,1,2 \ldots$ (i.e., for more than $2 n$ values) thus must be true for all complex $x$.

As mentioned in the introduction, there exist also other combinatorial proofs of the ${ }_{3} \phi_{2}$ summation. Among the references we have listed, Yee's paper [21] is remarkable as the proof there establishes the full $q$-Pfaff-Saalschütz summation at once and no appeal to a polynomial argument is needed.

3.2. $(a ; q)$-Extension of the alpha-parameter model. In [6], Goldman and Haglund introduced generalized rook models, called $i$-creation model and alpha-parameter model, which we briefly introduce first.

Given a board $B$, a file placement of $k$ rooks is a $k$-subset of $B$ such that no two cells lie in the same column, that is, there can be two or more rooks in the same row, but each column contains at most one rook. Let $\mathcal{F}_{k}(B)$ denote the set of all $k$-file placements. Given a Ferrers 
board $B$ and a file placement $P \in \mathcal{F}_{k}(B)$, we assign weights to the rows containing rooks as follows. If there are $u$ rooks in a given row, then the weight of this row is

$$
\begin{cases}1 & \text { if } 0 \leq u \leq 1 \\ \alpha(2 \alpha-1)(3 \alpha-2) \cdots((u-1) \alpha-(u-2)), & \text { if } u \geq 2\end{cases}
$$

The weight of a placement $P, w t(P)$, is the product of the weights of all the rows. Then for a Ferrers board $B$, set

$$
r_{k}^{(\alpha)}(B)=\sum_{P \in \mathcal{F}_{k}(B)} w t(P)
$$

Note that for $\alpha=0, r_{k}^{(0)}(B)$ reduces to the original rook number. If $\alpha$ is a positive integer $i$, $r_{k}^{(i)}(B)$ is the $i$-creation rook number which counts the number of $i$-creation rook placements of $k$ rooks on $B$. The $i$-creation rook placement is defined as follows: we first choose the columns to place the rooks. Then as we place rooks from left to right, each time a rook is placed, $i$ new rows are created drawn to the right end and immediately above where the rook is placed.

In this setting Goldman and Haglund [6] proved the $\alpha$-factorization theorem: given a Ferrers board $B=B\left(b_{1}, \ldots, b_{n}\right)$,

$$
\prod_{j=1}^{n}\left(z+b_{j}+(j-1)(\alpha-1)\right)=\sum_{k=0}^{n} r_{k}^{(\alpha)}(B) z(z+\alpha-1) \cdots(z+(n-k-1)(\alpha-1)) .
$$

Furthermore, Goldman and Haglund defined a $q$-analogue of $r_{k}^{(\alpha)}(B)$ by assigning $q$-weights to the cells in $B$. Here, we describe the $(a ; q)$-extension of their result which involves the use of the extra variable $a$ in the weights of the cells.

Given a Ferrers board $B$ and a rook placement $P \in \mathcal{N}_{k}(B)$, for each cell $c \in B$, let $v(c)$ be the number of rooks strictly to the left of, and in the same row as $c$ and $r_{c}(P)$ be the number of rooks in the north-west region of $c$. Then define the weight of $c$ to be

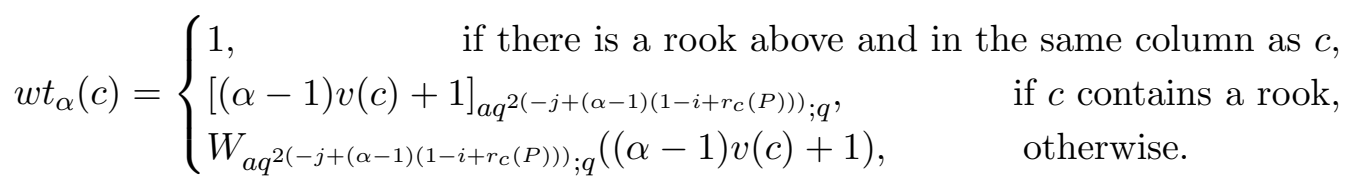

The weight of the rook placement $P$ is defined to be the product of the weights of all cells:

$$
w t_{\alpha}(P)=\prod_{c \in B} w t_{\alpha}(c) .
$$

We define an $(a ; q)$-analogue of $r_{k}^{(\alpha)}(B)$ by setting

$$
r_{k}^{(\alpha)}(a, q ; B)=\sum_{P \in \mathcal{F}_{k}(B)} w t_{\alpha}(P) .
$$

With this $r_{k}^{(\alpha)}(a, q ; B)$, we can also prove an $(a ; q)$-analogue of the $\alpha$-factorization theorem.

Theorem 3.7. For any Ferrers board $B=B\left(b_{1}, b_{2}, \ldots, b_{n}\right)$, we have

$$
\prod_{j=1}^{n}\left[z+b_{j}+(j-1)(\alpha-1)\right]_{a q^{-2\left(b_{j}+(j-1)(\alpha-1)\right)} ; q}
$$




$$
=\sum_{k=0}^{n} r_{n-k}^{(\alpha)}(a, q ; B) \prod_{i=1}^{k}[z+(i-1)(\alpha-1)]_{a q^{-2(i-1)(\alpha-1)} ; q^{\prime}} .
$$

Proof. Let us extend the board by attaching $z$ rows of width $n$ below the board $B$, denoted by $B_{z}$, and compute

$$
\sum_{P \in \mathcal{F}_{n}\left(B_{z}\right)} w t_{\alpha}(P)
$$

in two different ways. The left-hand side of (3.8) is the result of computing the above weight sum columnwise, and the right-hand side can be obtained by computing the weight of the cells in $B$ and and the cells in the extended part separately. For the details, see [20].

Remark 3.8. In [19], the authors have constructed a general rook theory model utilizing an augmented rook board which can be specialized to all the known rook theory models. The product formula in Theorem 3.7 was also obtained in [19, (4.17)] but by using a different approach.

In the case $\alpha=2$ and the board is of the staircase shape $S t_{n}=B(0,1,2, \ldots, n-1)$, $r_{k}^{(2)}\left(a, q ; S t_{n}\right)$ has a closed form expression (see [18]).

$$
r_{k}^{(2)}\left(a, q ; S t_{n}\right)=q^{-\left(\begin{array}{c}
n+k \\
2
\end{array}\right)+k(k+2)}\left[\begin{array}{c}
n+k-1 \\
2 k
\end{array}\right] \prod_{q=1}^{k}[2 j-1]_{q} \frac{\left(a q ; q^{-2}\right)_{n-k}\left(a q^{1-2 n} ; q^{2}\right)_{k}}{\left(a q ; q^{-4}\right)_{n}} .
$$

We now give combinatorial proofs of two special ${ }_{4} \phi_{3}$ summations.

\section{Proposition 3.9.}

$$
\frac{\left(q^{z+2}, a^{-1} q^{2-z} ; q^{2}\right)_{n}}{\left(q, a^{-1} q^{3} ; q^{2}\right)_{n}}=\sum_{k=0}^{n} \frac{\left(q^{-n},-q^{-n}, q^{z+1}, a^{-1} q^{1-z} ; q\right)_{k}}{\left(q, q^{-2 n}, a^{-1 / 2} q^{3 / 2},-a^{-1 / 2} q^{3 / 2} ; q\right)_{k}} q^{k}
$$

and,

$$
\frac{\left(q^{z+2}, a q^{z-2 n} ; q^{2}\right)_{n}}{\left(q^{z+1}, a q^{z-n} ; q\right)_{n}}=\sum_{k=0}^{n} \frac{\left(q^{-n}, q^{n+1}, a^{1 / 2} q^{-n-1 / 2},-a^{1 / 2} q^{-n-1 / 2} ; q\right)_{k}}{\left(q,-q, q^{-z-n}, a q^{z-n} ; q\right)_{k}} q^{k} .
$$

Proof. If we use the closed form expression for $r_{n-k}^{(2)}\left(a, q ; S t_{n}\right)$ of (3.9) in (3.8) for $b_{j}=j-1$, we get

$$
\begin{aligned}
& \frac{\left(q^{z} ; q^{2}\right)_{n}}{(1-q)^{n}} \frac{\left(a q^{z} ; q^{-2}\right)_{n}}{\left(a q ; q^{-4}\right)_{n}} q^{-n(z+1)}= \\
& \sum_{k=0}^{n} q^{-k(z+1)} \frac{(q ; q)_{2 n-k-1}}{(q ; q)_{2 n-2 k}(q ; q)_{k-1}} \frac{\left(q ; q^{2}\right)_{n-k}}{(1-q)^{n-k}} \frac{\left(a q ; q^{-2}\right)_{k}\left(a q^{1-2 n} ; q^{2}\right)_{n-k}}{\left(a q ; q^{-4}\right)_{n}} \frac{\left(q^{z} ; q\right)_{k}}{(1-q)^{k}} \frac{\left(a q^{z} ; q^{-1}\right)_{k}}{\left(a q ; q^{-2}\right)_{k}}
\end{aligned}
$$

which after some elementary manipulations simplifies to (3.10).

Similarly, (3.11) is the result of simplifying

$$
\prod_{j=1}^{n}[z+2(j-1)]_{a q^{-4(j-1)} ; q}=\sum_{k=0}^{n} r_{k}^{(2)}\left(a, q ; S t_{n}\right) \prod_{i=1}^{n-k}[z+i-1]_{a q^{-2(i-1)} ; q},
$$

after replacing $r_{k}^{(2)}\left(a, q ; S t_{n}\right)$ by the closed form expression in (3.9). 
The summation in (3.10) is equivalent (up to an obvious substitution of variables) to Jain's ${ }_{4} \phi_{3}$ summation (1.2) mentioned in the introduction.

The summation in (3.11) can be also verified by the following terminating $q$-analogue of Whipple's ${ }_{3} F_{2}$ sum [5. (II.19)],

$$
{ }_{4} \phi_{3}\left[\begin{array}{c}
q^{-n}, q^{n+1}, C,-C \\
E, C^{2} q / E,-q
\end{array} ; q, q\right]=\frac{\left(E q^{-n}, E q^{n+1}, C^{2} q^{1-n} / E, C^{2} q^{n+2} / E ; q^{2}\right)_{\infty}}{\left(E, C^{2} q / E ; q\right)_{\infty}} q^{\left(\begin{array}{c}
n+1 \\
2
\end{array}\right)},
$$

where we take $C=a^{1 / 2} q^{-n-1 / 2}$ and $E=q^{-z-n}$, and apply

$$
\begin{aligned}
& \frac{\left(q^{-z-2 n}, q^{1-z}, a q^{z-2 n}, a q^{z+1} ; q^{2}\right)_{\infty}}{\left(q^{-z-n}, a q^{z-n} ; q\right)_{\infty}} q^{\left(\begin{array}{c}
n+1 \\
2
\end{array}\right)} \\
= & \frac{\left(q^{-z-2 n} ; q^{2}\right)_{n}}{\left(q^{-z-n} ; q\right)_{n}} \frac{\left(q^{-z}, q^{1-z} ; q^{2}\right)_{\infty}}{\left(q^{-z} ; q\right)_{\infty}} \frac{\left(a q^{z-2 n} ; q^{2}\right)_{n}}{\left(a q^{z-n} ; q\right)_{n}} \frac{\left(a q^{z}, a q^{z+1} ; q^{2}\right)_{\infty}}{\left(a q^{z} ; q\right)_{\infty}} q^{\left(\begin{array}{c}
n+1 \\
2
\end{array}\right)} \\
= & \frac{\left(q^{z+2} ; q^{2}\right)_{n}}{\left(q^{z+1} ; q\right)_{n}} \frac{\left(a q^{z-2 n} ; q^{2}\right)_{n}}{\left(a q^{z-n} ; q\right)_{n}} .
\end{aligned}
$$

The two summations in (3.10) and (3.11) are actually equivalent to each other; one follows from the other by reversing the sum (i.e., substituting the summation index $k \mapsto n-k$ ).

3.3. $(a ; q)$-Rook theory for matchings. Haglund and Remmel [11] extended the rook theory by considering partial matchings as opposed to considering partial permutations in the original rook theory, and for which they consider the shifted board $B_{2 n}$ pictured in Figure 1.



Figure 1. $B_{2 n}$.

For each perfect matching $M$ of $K_{2 n}$ consisting of $n$ pairwise vertex disjoint edges in $K_{2 n}$, where $K_{2 n}$ is the complete graph on the set of vertices $\{1,2, \ldots, 2 n\}$, let

$$
P_{M}=\{(i, j) \mid i<j \text { and }\{i, j\} \in M\},
$$

where $(i, j)$ denotes the square in row $i$ and column $j$ of $B_{2 n}$ according to the labeling of rows and columns pictured in Figure 1. A rook placement in $B_{2 n}$ is defined to be a subset of some $P_{M}$ for a perfect matching $M$ of $K_{2 n}$.

Given a board $B \subseteq B_{2 n}$, we let $\mathcal{M}_{k}(B)$ denote the set of $k$ element rook placements in $B$. In this setting, we let $B\left(a_{1}, a_{2}, \ldots, a_{2 n-1}\right)$ denote the following set of cells in $B_{2 n}$ :

$$
B\left(a_{1}, a_{2}, \ldots, a_{2 n-1}\right)=\left\{(i, i+j) \mid 1 \leq i \leq 2 n-1,1 \leq j \leq a_{i}\right\} .
$$

It is called a shifted Ferrers board if $2 n-1 \geq a_{1} \geq a_{2} \geq \cdots \geq a_{2 n-1} \geq 0$ and the nonzero entries of $a_{i}$ 's are strictly decreasing. A rook in $(i, j)$ with $i<j$ in a rook placement cancels 
all cells $(i, s)$ in $B_{2 n}$ with $i<s<j$ and all cells $(t, j)$ and $(t, i)$ with $t<i$. See Figure 2 for a specific example of a shifted Ferrers board and the cells being cancelled by a rook on the shifted board $B_{8}$.


Figure 2. The shifted Ferrers board $B=(7,5,4,2,0,0,0) \subseteq B_{8}$, and the cells cancelled by a rook in $(4,7)$ on $B_{8}$.

Definition 3.10. Given a shifted Ferrers board $B=B\left(a_{1}, \ldots, a_{2 n-1}\right) \subseteq B_{2 n}$ and a rook placement $P \in \mathcal{M}_{k}(B)$, define

$$
m_{k}(a, q ; B)=\sum_{P \in \mathcal{M}_{k}(P)} w t_{m}(P),
$$

where

$$
w t_{m}(P)=\prod_{(i, j) \in U_{B}(P)} w_{a ; q}\left(\hat{i}+\hat{j}-1-2 r_{(i, j)}(P)-s_{(i, j)}(P)\right),
$$

$U_{B}(P)$ denote the set of cells in $B$ which are neither cancelled by rooks nor contain any rooks in $P, r_{(i, j)}(P)$ is the number of rooks in $P$ positioned south-east of $(i, j)$ such that the two columns cancelled by those rooks are to the right of the column $j, s_{(i, j)}(P)$ is the number of rooks in $P$ which are in the south-east region of $(i, j)$ such that only one cancelled column is to the right of column $j$, and $\hat{i}:=2 n-i$.

We also have a product formula involving $m_{k}(a, q ; B)$, which is an $(a ; q)$-analogue of the product formula proved by Haglund and Remmel [11].

Theorem 3.11. Given a shifted Ferrers board $B=B\left(a_{1}, \ldots, a_{2 n-1}\right) \subseteq B_{2 n}$, we have

$$
\prod_{i=1}^{2 n-1}\left[z+a_{2 n-i}-2 i+2\right]_{a q^{2\left(2 i-2-a_{2 n-i}\right) ; q}}=\sum_{k=0}^{n} m_{k}(a, q ; B) \prod_{j=1}^{2 n-1-k}[z-2 j+2]_{a q^{4 j-4} ; q} .
$$
form

In the case of the full board $B_{2 n}=B(2 n-1,2 n-2, \ldots, 2,1), m_{k}\left(a, q ; B_{2 n}\right)$ has a closed

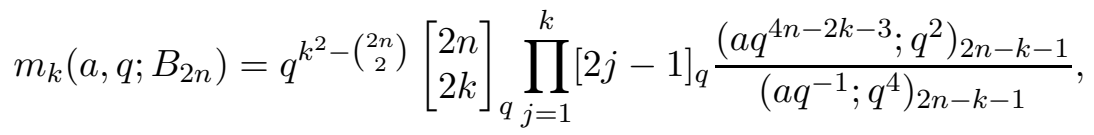


which can be verified by the following recursion

$$
\begin{aligned}
& m_{k}\left(a, q ; B_{N}\right) \\
& =[N-2 k+1]_{a q^{2(N-3)} ; q} m_{k-1}\left(a, q ; B_{N-1}\right)+W_{a q^{2(N-3)} ; q}(N-2 k-1) m_{k}\left(a, q ; B_{N-1}\right) .
\end{aligned}
$$

Replacing $m_{k}(a, q ; B)$ in (3.12) for $a_{i}=2 n-i$ by (3.13) gives a special case of the $q$-PfaffSaalschütz sum.

\section{Proposition 3.12.}

$$
\frac{\left(q^{z-n+\frac{3}{2}}, a q^{z-\frac{1}{2}} ; q\right)_{n}}{\left(q^{z-2 n+2}, a q^{z+n-\frac{1}{2}} ; q\right)_{n}}=\sum_{k=0}^{n} \frac{\left(q^{-n}, q^{-n+\frac{1}{2}}, q^{\frac{5}{2}-2 n} / a ; q\right)_{k}}{\left(q, q^{z-2 n+2}, q^{2-2 n-z} / a ; q\right)_{k}} q^{k}
$$

Proof. Putting the closed form expression for $m_{k}\left(a, q ; B_{2 n}\right)$ in (3.12) for $a_{i}=2 n-i$ gives

$$
\begin{aligned}
& \frac{\left(q^{z+1} ; q^{-1}\right)_{2 n-1}\left(a q^{z-1} ; q\right)_{2 n-1}}{(1-q)^{2 n-1}\left(a q^{-1} ; q^{2}\right)_{2 n-1}} \\
& =\sum_{k=0}^{n} q^{2 k(k-2 n+1)+k z} \frac{(q ; q)_{2 n}}{(q ; q)_{2 k}(q ; q)_{2 n-2 k}} \frac{\left(q ; q^{2}\right)_{k}}{(1-q)^{k}} \frac{\left(a q^{z}, a q^{4 n-2 k-3} ; q^{2}\right)_{2 n-k-1}}{\left(a q, a q^{-1} ; q^{4}\right)_{2 n-k-1}} \frac{\left(q^{z} ; q^{-2}\right)_{2 n-k-1}}{(1-q)^{2 n-k-1}}
\end{aligned}
$$

which simplifies to

$$
\frac{\left(q^{-z-2}, a q^{z-2} ; q\right)_{2 n}}{\left(q^{-z-2}, a q^{z-2} ; q^{2}\right)_{2 n}} q^{n(2 n-1)}=\sum_{k=0}^{n} \frac{\left(q^{-2 n}, q^{1-2 n}, q^{5-4 n} / a ; q^{2}\right)_{k}}{\left(q^{2}, q^{z-4 n+4}, q^{4-4 n-z} / a ; q^{2}\right)_{k}} q^{2 k} .
$$

The identitiy can now be obtained by replacing $q^{2} \rightarrow q$ and $z / 2 \rightarrow z$.

Remark 3.13. The identity (3.14) (proved combinatorially) is actually the

$$
A=q^{-n+\frac{1}{2}}, \quad B=q^{\frac{5}{2}-2 n} / a, \quad \text { and } C=q^{z-2 n+2}
$$

special case of the $q$-Pfaff-Saalschütz sum (3.7).

\section{REFERENCES}

[1] G. E. Andrews, "Partitions", in Combinatorics: ancient and modern, (R. Wilson et al., eds.) Oxford University Press, Oxford (2013), 205-229.

[2] G. E. Andrews and D. M. Bressoud, "Identities in combinatorics III: further aspects of ordered set sorting", Discrete Math. 49 (1984), 223-236.

[3] W. N. Bailey, "On the sum of a terminating ${ }_{3} F_{2}(1)$ ", Quart. J. Math., Oxford Ser. (2) 4 (1953), $237-240$.

[4] F. Butler, M. Can, J. Haglund and J. B. Remmel, "Rook theory notes", book project http://www.math.ucsd.edu/ remmel/files/Book.pdf

[5] G. Gasper and M. Rahman, Basic hypergeometric series, second edition, Encyclopedia of Mathematics and Its Applications 96, Cambridge University Press, Cambridge, 2004.

[6] J. Goldman and J. Haglund, "Generalized rook polynomials", J. Combin. Theory Ser. A 91 (2000), 509-530.

[7] J. R. Goldman, J. T. Joichi and D. E. White, "Rook theory. IV. Orthogonal sequences of rook polynomials", Stud. Appl. Math 56 (1976/1977), 267-272.

[8] I. P. Goulden, "A bijective proof of the q-Saalschütz theorem", Discrete Math. 57 (1985), 39-44.

[9] V. W. Guo and J. Zeng, "A combinatorial proof of a symmetric q-Pfaff-Saalschütz identity", Electron. J. Combin. 12 (2005), \#N2.

[10] J. Haglund, "Rook theory and hypergeometric series", Adv. in Appl. Math. 17 (1996), 408-459.

[11] J. Haglund and J. B. Remmel, "Rook theory for perfect matchings", Adv. Appl. Math. 27 (2001), $438-481$.

[12] V. K. Jain, "Some transformations of basic hypergeometric functions, II", SIAM J. Math. Anal. 12 (1981), 957-961.

[13] I. Kaplansky and J. Riordan, "The problem of the rooks and its applications", Duke Math. J. 13 (1946), 259-268. 
[14] T. Mansour and M. Schork, "Commutation Relations, Normal Ordering, and Stirling Numbers", Chapman and Hall/CRC Press, 2015.

[15] G. Nyul and G. Rácz, "The r-Lah numbers", Discrete M. 338 (2015), 1660-1666.

[16] M. J. Schlosser, "Elliptic enumeration of nonintersecting lattice paths", J. Combin. Theory Ser. A 114 (2007), 505-521.

[17] M. J. Schlosser, "A noncommutative weight-dependent generalization of the binomial theorem", preprint arXiv: 1106.2112.

[18] M. J. Schlosser and M. Yoo, "Elliptic rook and file numbers", preprint arXiv:1512.01720.

[19] M. J. Schlosser and M. Yoo, "An elliptic extension of the general product formula for augmented rook boards", Europ. J. Combin., to appear; preprint arXiv:1601.07834.

[20] M. J. Schlosser and M. Yoo, "Elliptic extensions of the alpha-parameter model and the rook model for matchings", preprint arXiv:1606.02928.

[21] Ae-Ja Yee, "Combinatorial proofs of identities in basic hypergeometric series", Europ. J. Combin. (5) 29 (2008), 1365-1375.

[22] D. Zeilberger, "A $q$-Foata proof of the $q$-Saalschütz identity", Europ. J. Combin. 8 (1987), 461-463.

Fakultät für Mathematik, Universität Wien, Oskar-Morgenstern-Platz 1, A-1090 Vienna, AUSTRIA

E-mail address: michael.schlosser@univie.ac.at

$U R L:$ http://www.mat.univie.ac.at/〜schlosse

Fakultät für Mathematik, Universität Wien, Oskar-Morgenstern-Platz 1, A-1090 Vienna, AUstria

E-mail address: meesue.yoo@univie.ac.at 